\title{
El codiseño educativo haciendo uso de las TIC en educación superior: una revisión sistemática de literatura
}

\author{
Learning co-design and the use of ICT in higher education: a systematic literature review
}

Jennifer Saray Santana Martel; santanamartel@hotmail.com

D Adolfina Pérez i Garcias; fina.perez@uib.es

Universidad de las Islas Baleares (España)

\section{Resumen}

Esta revisión sistemática de literatura (RSL) presenta el análisis bibliométrico de los documentos seleccionados, la forma en que los profesores y los estudiantes co-diseñan dentro del currículo de una asignatura haciendo uso las tecnologías de la información y del conocimiento (TIC) en la educación superior (ES), así como los beneficios y desafíos que surgen en este proceso. Para filtrar los artículos se seleccionaron cuatro criterios de inclusión: fuente (bases de datos Scopus y Redalyc), idioma (español e inglés), periodo de tiempo (2014-2019) y contexto (codiseño entre profesores y estudiantes haciendo uso de la tecnología dentro del marco de una asignatura en instituciones de ES). Los resultados muestran que no existe un proceso unificado para co-diseñar haciendo uso de las TIC en ES. Además, el co-diseño educativo (CE) muestra varios desafíos, sin embargo, son esos mismos, los que hacen que la asociación entre profesor y alumnado sea beneficiosa. No obstante, para superar estos obstáculos, se sugirieron algunos requisitos a cumplir para el buen funcionamiento del CE. Se recomienda, diseñar un modelo de CE mediado por tecnología educativa que establezca secuencias didácticas, así como, indagar sobre cómo co-diseñar la evaluación entre profesor y estudiante haciendo uso de las TIC.

Palabras clave: codiseño educativo, TIC, Educación superior, revisión sistemática de literatura

\section{Abstract}

This systematic literature review (SLR) presents the biliometric characteristics of the articles selected, the way staff and students co-design within the curriculum of a subject using information and communication technology (ICT) in higher education, and the benefits and challenges of this process. To crib the articles, we selected four inclusion criteria: source (Scopus and Redalyc database), language (Spanish and English), period of time (2014-2019), and context (staff-student co-design within the curriculum of a subject using ICT in higher education). Results shows that there is not a unified method to be followed in order to co-design in higher education. Besides, learning co-design seems to have several challenges, nonetheless, those same challenges seem to be also the ones that makes the staff-student partnership beneficial. However, as to overcome these obstacles and accomplish the learning process successfully, some requirements are suggested by the primary studies' authors. As this research shows, there is still a need to keep investigating on how learning codesign takes place while using ICT in higher education. Therefore, a learning co-design model that uses educational technology through didactic sequences should be design. Furthermore, an inquiry on how staff-student co-design assessment while using ICT should be carried out.

Keywords: learning co-design, ICT, higher education, systematic learning review 


\section{INTRODUCCIÓN}

Los estudiantes de educación superior del siglo XXI, denominados "la Generación P" debido a su carácter participativo en todos los ámbitos de su vida (Kalantzis y Cope, 2010), han sido subestimados por el sistema educativo. Actualmente, la participación de los alumnos se ve limitada a su compromiso, retención, creación de comunidades de aprendizaje, desarrollo de habilidades de empleo... (Kaminskiene et al., 2020), mas no se les hace partícipes en la toma de decisiones en cuanto a su proceso educativo. Por consiguiente, surge la necesidad de que se produzca un cambio de paradigma en el proceso de enseñanza-aprendizaje.

El diseño participativo ha sido la manera de implicar a los usuarios (profesores y estudiantes) como socios (Sanders y Stappers, 2008, p. 6) y, en los últimos años, las nociones de co-diseño y cocreación han venido evolucionado dentro de esta área (Sanders y Stappers, 2008). Para Sanders y Stappers, (2008), el co-diseño abarca todo el proceso de diseño, mientras que la co-creación está delimitado a un acto de creatividad dentro del co-diseño. Sin embargo, siendo ambos conceptos ejemplos del diseño participativo, se diferencian en la forma en la que se elige colaborar con los usuarios, dependiendo del contexto y de los objetivos que se tengan (Dollinger et al., 2019). Por ejemplo; se pueden asociar entre docentes, entre estudiantes, docentes y alumnos (Könings et al., 2020; Manrique-Losada et al., 2020; Mercer-mapstone et al., 2019; Pee, 2020), universidad y empresas (Silva y Rossi, 2018) y entre empresa privada o pública, profesores y estudiantes (HanniVaara et al., 2019; Jussila et al., 2019; Mcfaul et al., 2020)

Para Bovill et al. (2016) la co-creación ocurre cuando los participantes trabajan de manera colaborativa para crear componentes del currículo y/o del enfoque metodológico. Además, Bovill et al. (2016) distinguen entre la co-creación del currículo y la co-creación en el currículo; mientras que en la primera, se co-diseña un programa o curso antes de que este comience, en la segunda, se co-diseña durante el desarrollo del mismo. Según Kaminskiene et al. (2020) es esta última, la forma más recurrente de Co-diseño Educativo (CE) y es allí donde se descubren nuevos desafíos para todos los involucrados, ya que pueden usarse diferentes niveles de interacción, tales como los propuestos por Bovill y Bulley (2011) en los que proponen cuatro categorías que van en aumento de acuerdo con el nivel de participación en la toma de decisiones que los estudiantes adquieren; desde el control del docente hasta un considerable control por parte de los alumnos. Asimismo, los estudiantes generalmente asumen o el rol de asesor o co-investigador o codiseñador pedagógico o portavoz durante el proceso de co-creación (Bovill et al., 2016).

Por otro lado, el CE busca involucrar a los alumnos como socios de dos formas; en primer lugar, y predominantemente, en el aprendizaje, la enseñanza y la evaluación y, en segundo lugar, en el diseño curricular y la consultoría pedagógica (Healey et al., 2014). Por tanto, la implicación de los alumnos va a influir tanto en la toma de decisiones sobre cómo se desarrolla el aprendizaje y en qué tipo de tecnología satisface mejor sus necesidades educativas (Gros y López, 2016), como en la contribución al desarrollo de estrategias de autorregulación en el aprendizaje (Treasure-Jones et al., 2018). Además, la co-creación es beneficiosa en el proceso de enseñanza-aprendizaje 
debido a que se mejora el ambiente de aprendizaje, la motivación y la metacognición en los participantes directos así como la calidad del diseño educativo del curso o del currículo y la forma de enseñar (Könings et al., 2020).

Finalmente, es necesario apuntar que aunque la literatura y el interés en el CE en ES se está incrementando (Bovill et al., 2016; Deeley y Bovill, 2017; Doyle et al., 2019; Könings et al., 2020), los estudios que lo promueven no son suficientes, y los existentes, no incluyen información sobre el funcionamiento de esta alianza (Bryson, 2016). Además, la literatura que incorpora el CE y las tecnologías en ES, particularmente en estudios de grado, es limitada (Gros, 2019). Por lo que esta pesquisa pretende conocer la manera en la que los estudiantes y profesores co-diseñan en ES, específicamente haciendo uso de las TIC, de acuerdo a la literatura científica de los últimos seis años (2014-2019).

\section{MÉTODOS}

La RSL nos permite desarrollar un proceso investigativo que busca dar sentido a la documentación encontrada, así como una manera de dar respuesta a las preguntas acerca de lo que realmente funciona (Petticrew y Roberts, 2006), específicamente en el CE. Para ello, se ejecutó un protocolo debidamente definido, en el cual se siguieron los siguientes seis pasos adaptados de ZawackiRichter et al. (2020):
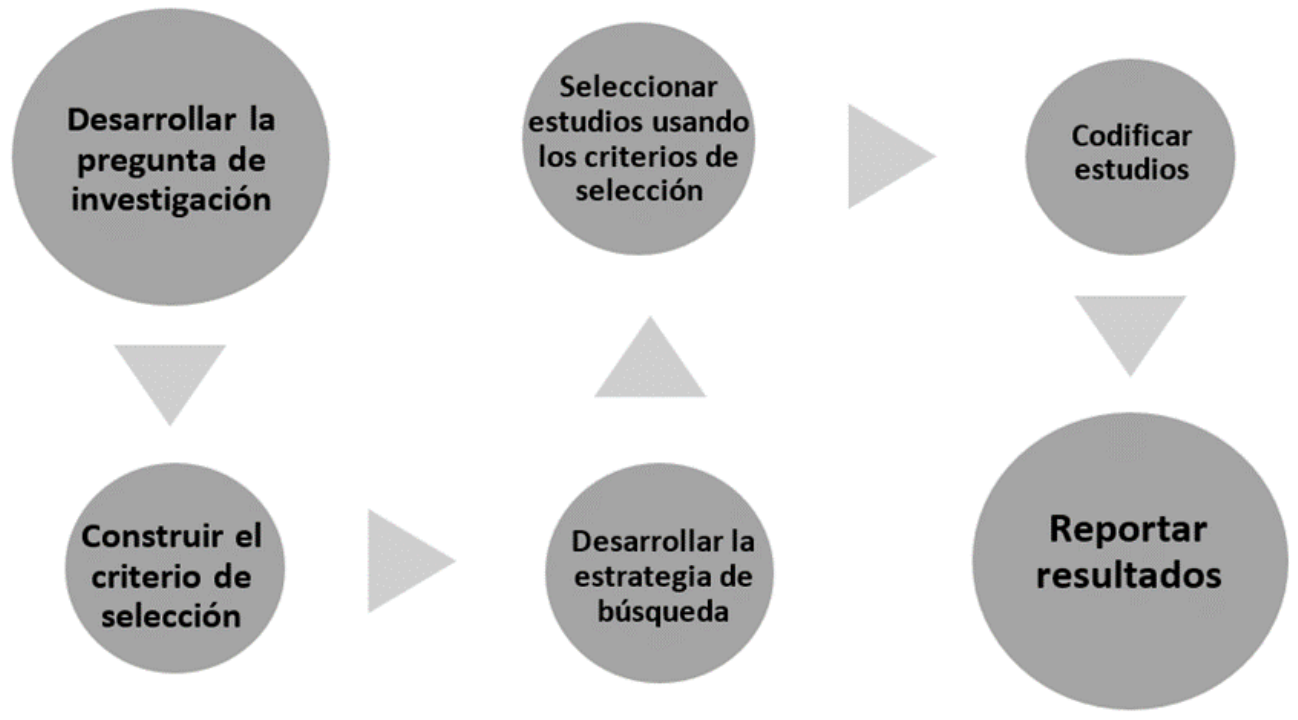

Figura 1. Procesos de la RSL 


\section{Preguntas de investigación:}

¿De qué manera los estudiantes y profesores co-diseñan haciendo uso de la tecnología en ES de acuerdo a la literatura científica de los últimos seis años (2014-2019)? Esta pregunta se desglosa en tres preguntas más concretas:

1. ¿Qué características bibliométricas presentan los estudios primarios (EP)?

2. ¿Cuáles son las características de las experiencias de co-diseño que se describen en los EP?

3. ¿Qué beneficios y desafíos se presentan durante el co-diseño entre profesor y estudiante haciendo uso de la tecnología en ES?

\section{Criterios de inclusión (Cl)}

Los criterios de inclusión de los EP:

- $\quad$ Cl-1 (Fuente): artículos publicados en las bases de datos Scopus y Redalyc.

- $\quad \mathrm{Cl}-2$ (Idioma): artículos publicados en español y/o en inglés.

- $\quad \mathrm{Cl}-3$ (Periodo de tiempo): publicados entre 2014 y 2019.

- Cl-4 (Contexto): co-diseño entre profesores y estudiantes haciendo uso de la tecnología dentro del marco de una asignatura en instituciones de ES.

\section{Conducta de la búsqueda}

La búsqueda de artículos de co-diseño en el ámbito de educación en general se condujo el día 11 de febrero de 2020, según se presenta en la tabla 1.

Tabla 1. Conducta de la búsqueda en la base de datos Scopus y Redalyc

\begin{tabular}{ccc}
\hline Base de datos & Búsqueda ejecutada & Resultados \\
\hline Scopus & "TITLEABS-KEY [Co-design OR Codesign OR Codiseño OR Co-diseño] AND & 556 \\
& TITLE-ABS-KEY [Education OR Educación] & 24 \\
\hline Redalyc & "Co-design OR Codesign OR Codiseño OR Co-diseño AND Education OR \\
& Educación" & \\
\hline
\end{tabular}

\section{Selección de estudios}

En primer lugar, se aplicó el Cl-1, Cl-2 y Cl-3, arrojando 192 artículos (176 de Scopus y 16 de Redalyc). En segundo lugar se aplicó el $\mathrm{Cl}-4$, para ello, se utilizó el programa Rayyan. Como resultado, se cribaron 23 artículos. Sin embargo, tras una profunda lectura de los EP, se descartaron 17 debido al no cumplimiento de algunos $\mathrm{Cl}-4$, en su mayoría debido a que el CE no 
se realizó dentro del currículo de una asignatura y/o no usaron las TIC para co-diseñar. En la figura 2 , se describe y resume el procedimiento de selección de artículos.

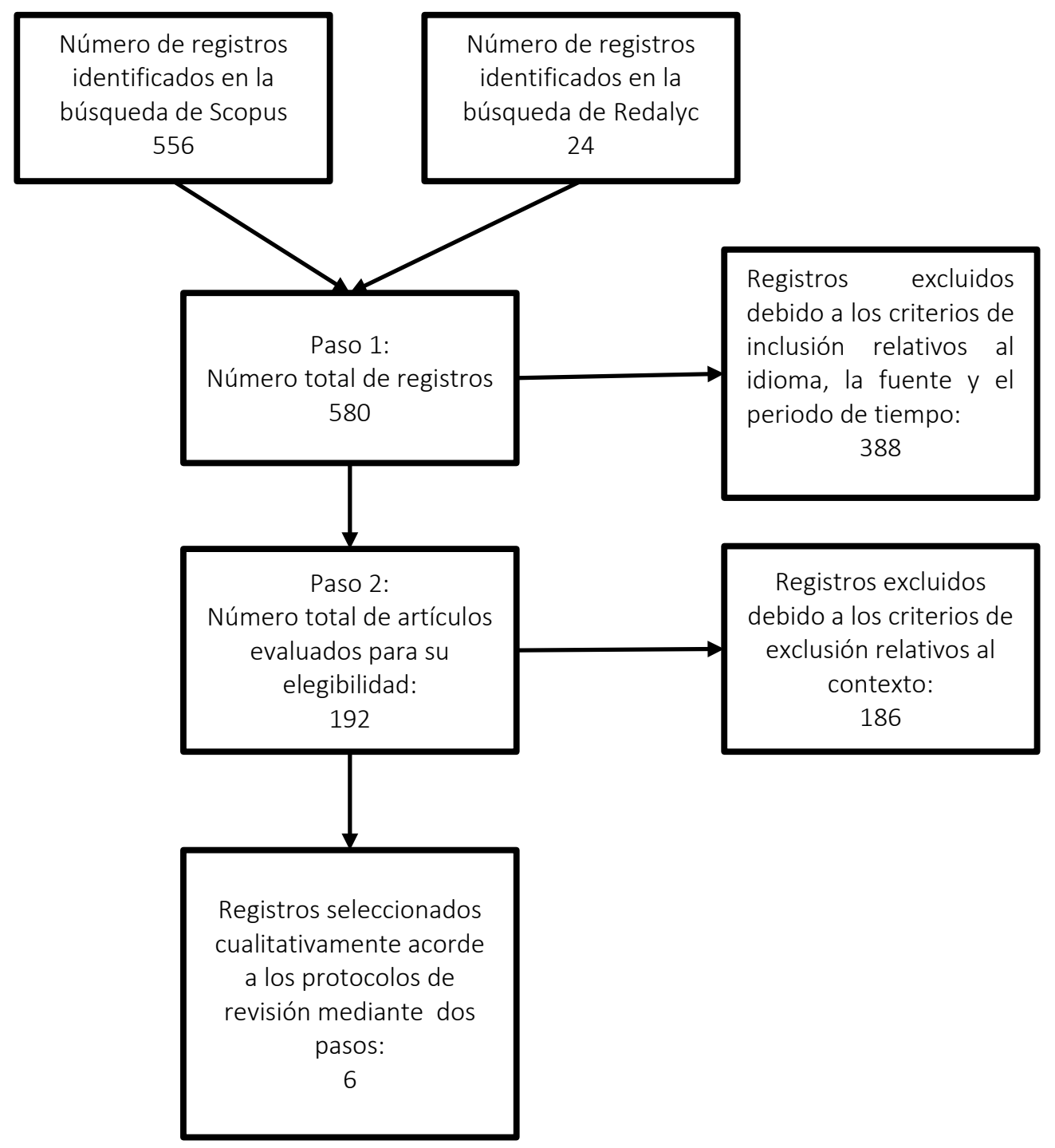

Figura 2. Diagrama de flujo del proceso de selección de artículos.

\section{Codificación de estudios y análisis de resultados}

Para el presente análisis se establecieron tres dimensiones: (1) análisis bibliométrico, (2) características de las experiencias de co-diseño, y (3) los beneficios y los desafíos de las mismas. Para el análisis bibliométrico, se distinguieron 6 categorías: año de publicación, número de citas incluidas, palabras clave, lugar de procedencia según el primer autor del artículo (país y continente), revistas donde fueron publicadas y tipo de artículo (investigativo, experiencia 
educativa...). Para su análisis, se utilizó la herramienta de Microsoft Excel y VOSviewer. Respecto a las características de co-diseño, se analizaron 8 categorías: tipo de co-diseño que presentan las experiencias, tipo de institución involucrada, tipo de asignatura donde se llevó a cabo la experiencia, tecnología utilizada, personas involucradas, conceptos estrechamente relacionados con el co-diseño educativo, metodologías asociadas al co-diseño y estrategias metodológicas utilizadas para co-diseñar. La tercera dimensión, por su parte, presenta dos categorías: beneficios y desafíos. Para la codificación de la segunda y tercera dimensión se usó Atlas.ti.

\section{RESULTADOS}

A continuación, se presentan los resultados de cada una de las preguntas de investigación:

\subsection{Características bibliométricas de los EP}

\section{Años de publicación}

El análisis bibliométrico referente a "años de publicación", muestra intervalos de crecimiento y decrecimiento. Durante el año 2014 y 2015 no se encontraron publicación, no obstante, el año 2016 fue el que obtuvo mayor cantidad de publicaciones, mientras que en los siguientes años (2017-2019) se produce un notorio descenso (17\%). Por lo que, se entiende que el año 2016 marca un punto de interés que se ha mantenido en los siguientes años.

Grafica 1. Año de publicación de los artículos seleccionados.

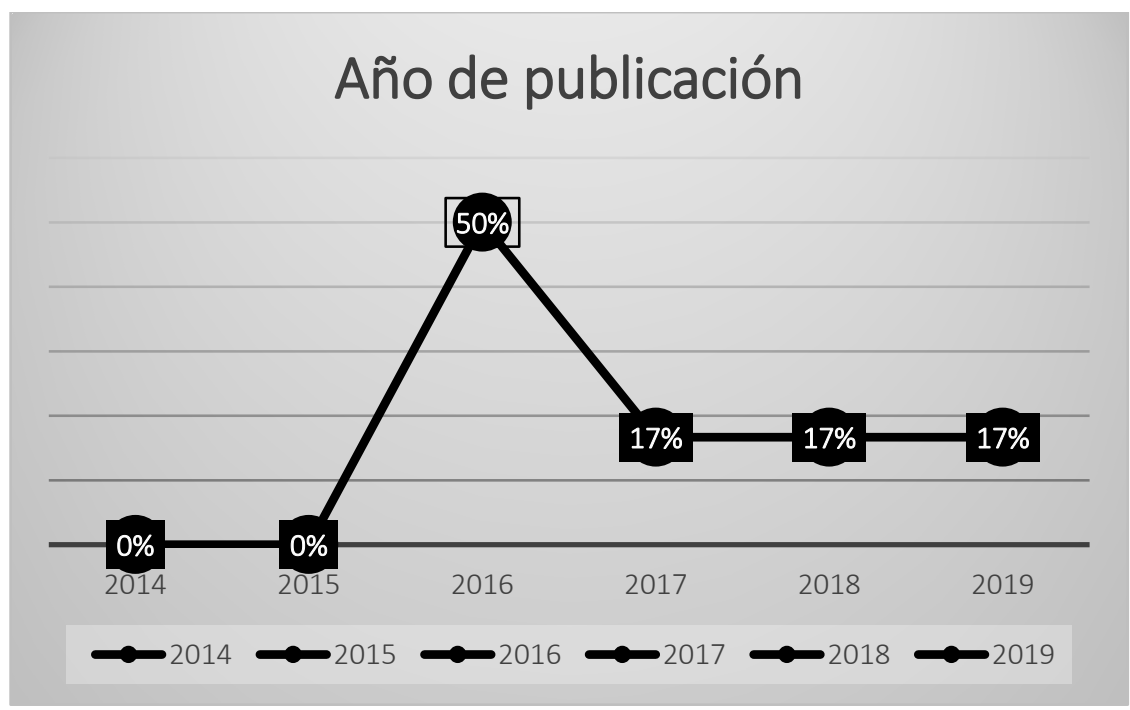




\section{Palabras clave}

A partir de las 32 palabras clave de los artículos se elaboró una red de co-ocurrencia de palabras clave generada en Vosviewer, logrando visualizar las conexiones existentes entre ellas. Esta red quedó dividida en seis grupos de palabras. Cada grupo presenta palabras conectadas entre sí, sin embargo, no se presentan conexiones entre los otros grupos como se resume en la tabla 2.

Tabla 2. Coocurrencia de palabras clave de los autores

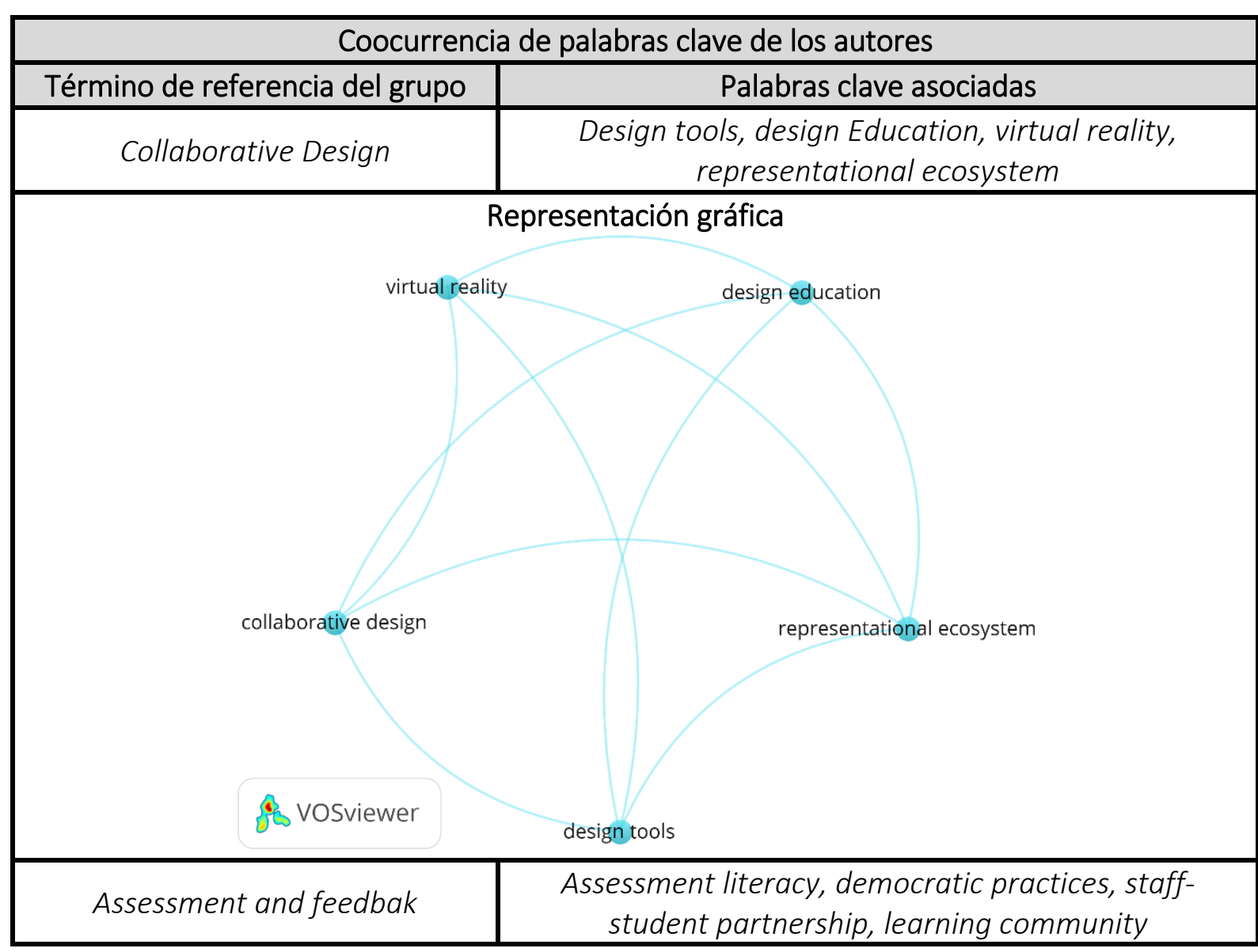




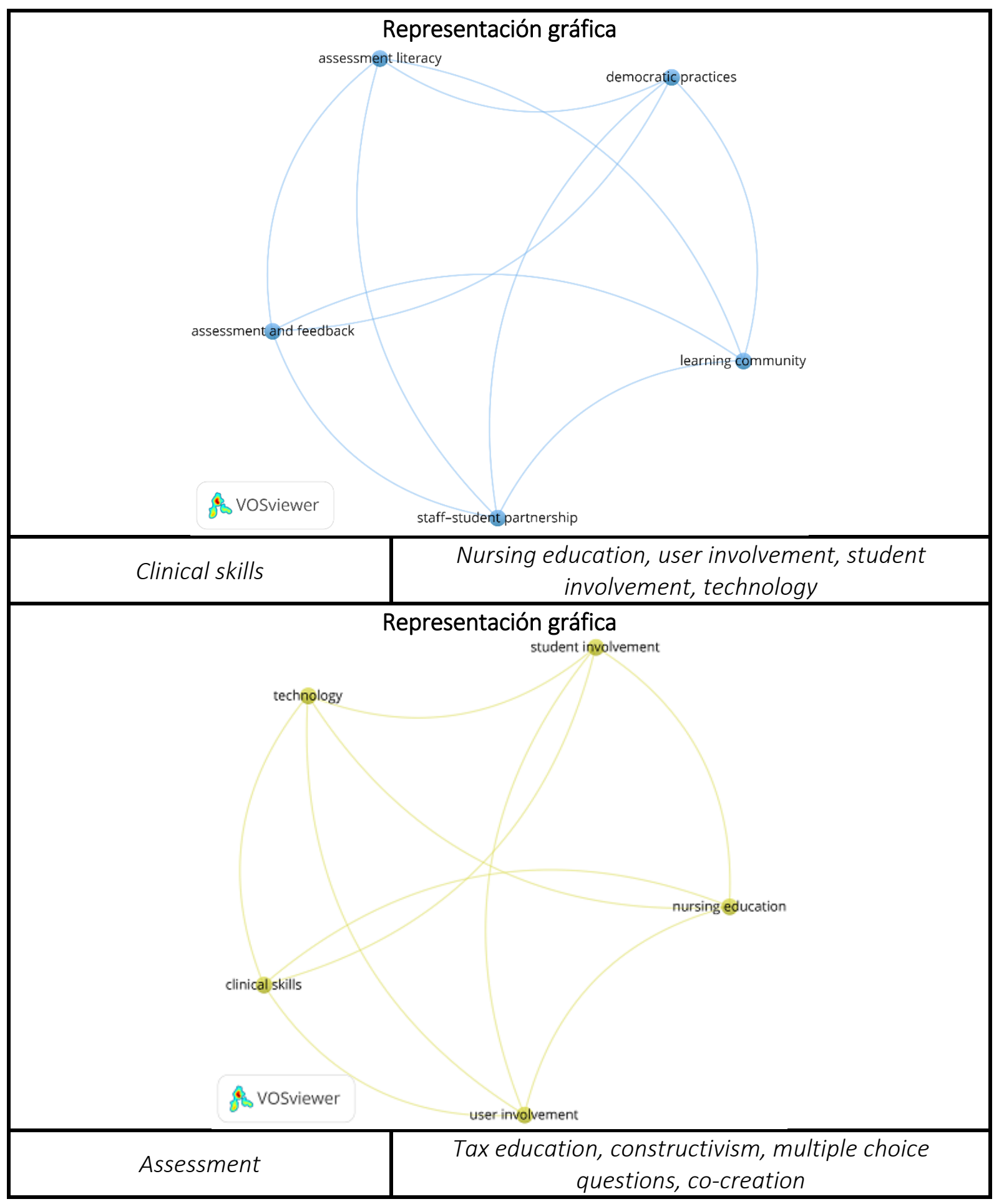




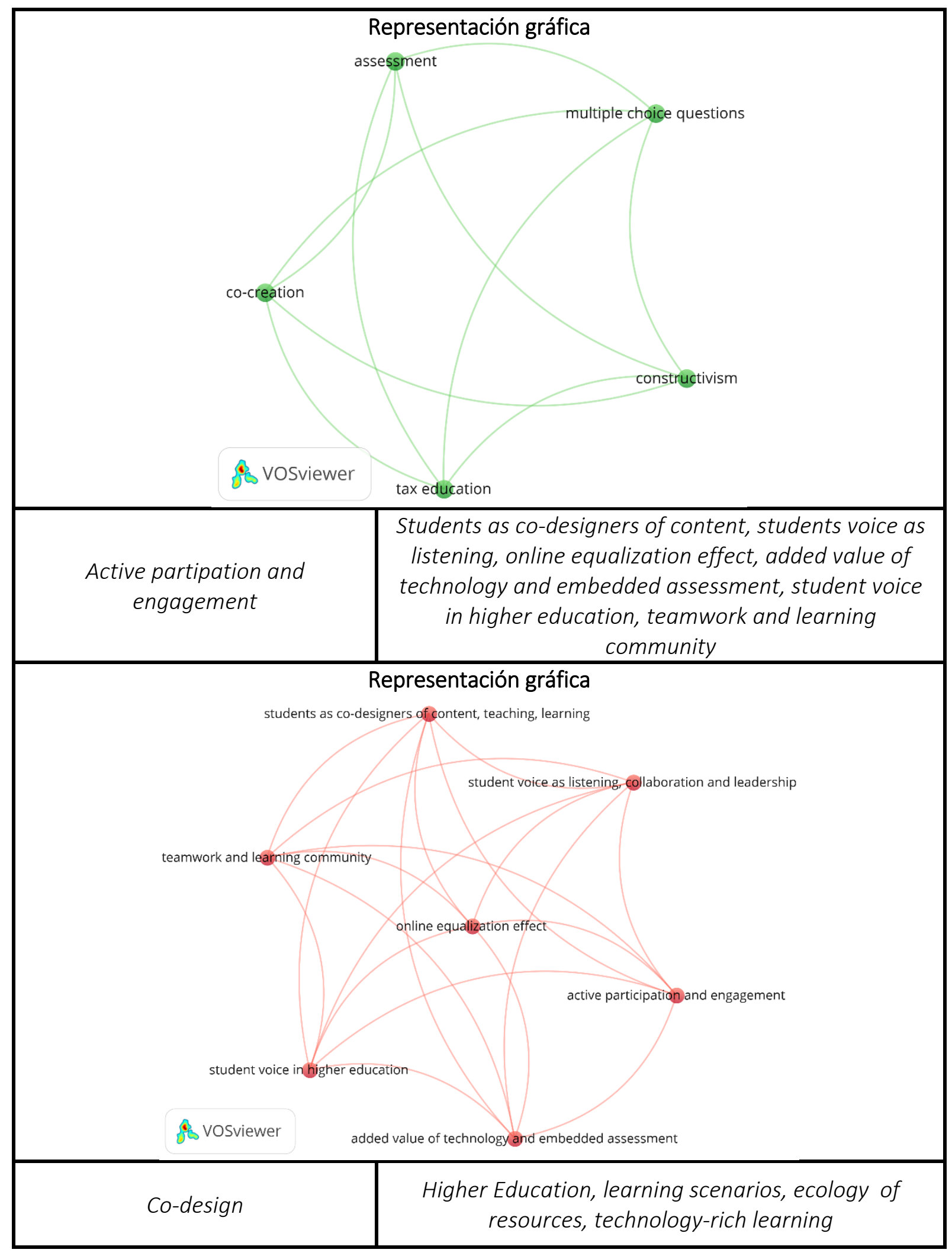




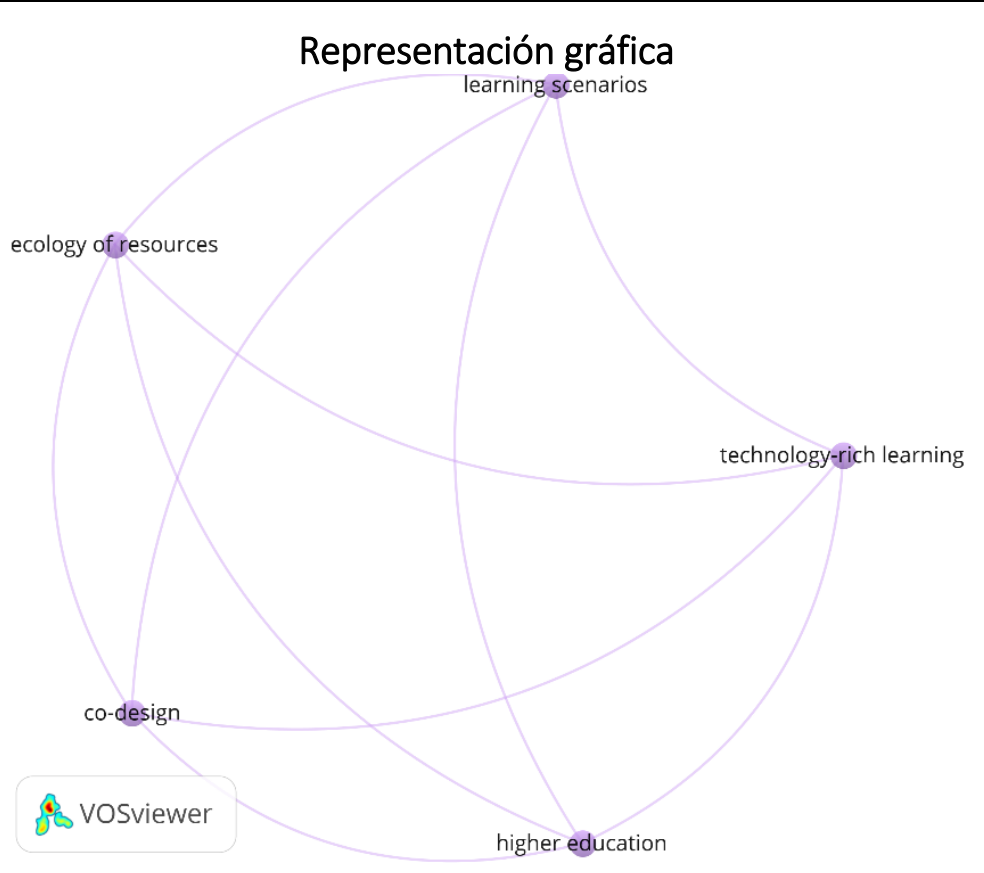

Número de citas incluidas, clasificación de revistas según Scopus, procedencia de los artículos y tipo de artículo

En la tabla 3 se presenta el impacto que los artículos seleccionados a día de hoy ${ }^{1}$, en el que se resalta la influencia del tercer estudio. No obstante, se observa que todos los artículos fueron publicados en revistas clasificadas en el cuartil 1 según Scopus. También, se evidencia que el continente predominante en el área de conocimiento sobre CE haciendo uso de las TIC es Europa con cuatro países de referencia. Asimismo, se resalta que todos los artículos presentan investigaciones sobre experiencias educativas, de estos, 3 son estudios exploratorios (Doyle et al., 2019; Gros y López, 2016; Haraldseid et al., 2016) y 3 estudios de caso (Blau y Shamir-Inbal, 2018; Deeley y Bovill, 2017; Dorta et al., 2016). 
Tabla 3. Artículos seleccionados: título, autor, editorial, tipo de artículo, país y continente según la afiliación del primer autor, clasificación de las revistas según Scopus y sus correspondientes citaciones

\begin{tabular}{|c|c|c|c|c|c|c|}
\hline $\begin{array}{l}\text { (Número del artículo) } \\
\text { Nombre del artículo }\end{array}$ & Autor & Editorial & $\begin{array}{l}\text { Tipo de } \\
\text { artículo }\end{array}$ & $\begin{array}{c}\text { País - } \\
\text { Continente }\end{array}$ & $\begin{array}{c}\text { Clasificación } \\
\text { Revistas Scopus }\end{array}$ & Citaciones \\
\hline $\begin{array}{c}\text { (1) Assessment co- } \\
\text { creation: an exploratory } \\
\text { analysis of opportunities } \\
\text { and challenges based on } \\
\text { student and instructor } \\
\text { perspectives }\end{array}$ & $\begin{array}{l}\text { Elaine Doyle, } \\
\text { Patrick } \\
\text { Buckley y } \\
\text { Joanne } \\
\text { Whelan }\end{array}$ & $\begin{array}{l}\text { Teaching in } \\
\text { Higher } \\
\text { Education }\end{array}$ & $\begin{array}{l}\text { Estudio } \\
\text { exploratorio }\end{array}$ & $\begin{array}{l}\text { Irlanda - } \\
\text { Europa }\end{array}$ & Q1 & 0 \\
\hline $\begin{array}{l}\text { (2) Digital technologies } \\
\text { for promoting "student } \\
\text { voice" and co-creating } \\
\text { learning experience in } \\
\text { an academic course }\end{array}$ & $\begin{array}{c}\text { Ina Blau y } \\
\text { Tamar } \\
\text { Shamir-Inbal }\end{array}$ & $\begin{array}{l}\text { Instructional } \\
\text { Science }\end{array}$ & $\begin{array}{l}\text { Estudio de } \\
\text { caso }\end{array}$ & Israel-Asia & Q1 & 8 \\
\hline $\begin{array}{c}\text { (3) Staff student } \\
\text { partnership in } \\
\text { assessment: enhancing } \\
\text { assessment literacy } \\
\text { through democratic } \\
\text { practices } \\
\end{array}$ & $\begin{array}{l}\text { Susan J. } \\
\text { Deeley y } \\
\text { Catherine } \\
\text { Bovill }\end{array}$ & $\begin{array}{l}\text { Assessment } \\
\text { and } \\
\text { Evaluation in } \\
\text { Higher } \\
\text { Education }\end{array}$ & $\begin{array}{l}\text { Estudio de } \\
\text { caso }\end{array}$ & $\begin{array}{l}\text { Reino } \\
\text { Unido- } \\
\text { Europa }\end{array}$ & Q1 & 29 \\
\hline $\begin{array}{l}\text { (4) Students as co- } \\
\text { creators of technology- } \\
\text { rich learning activities in } \\
\text { higher education }\end{array}$ & $\begin{array}{l}\text { Begoña Gros y } \\
\text { Marta López }\end{array}$ & $\begin{array}{l}\text { International } \\
\text { Journal of } \\
\text { Educational } \\
\text { Technology in } \\
\text { Higher } \\
\text { Education }\end{array}$ & $\begin{array}{l}\text { Estudio } \\
\text { exploratorio }\end{array}$ & $\begin{array}{l}\text { España- } \\
\text { Europa }\end{array}$ & Q1 & 5 \\
\hline $\begin{array}{l}\text { (5) A new } \\
\text { representational } \\
\text { ecosystem for design } \\
\text { teaching in the studio }\end{array}$ & $\begin{array}{l}\text { Tomás Dorta, } \\
\text { Gôkçe } \\
\text { Kinayoglu y } \\
\text { Sana } \\
\text { Boudhraâ }\end{array}$ & $\begin{array}{l}\text { Design } \\
\text { Studies }\end{array}$ & $\begin{array}{l}\text { Estudio de } \\
\text { caso }\end{array}$ & $\begin{array}{l}\text { Canadá- } \\
\text { Norte } \\
\text { América }\end{array}$ & Q1 & 2 \\
\hline $\begin{array}{c}\text { (6) How can students } \\
\text { contribute? A } \\
\text { qualitative study of } \\
\text { active student } \\
\text { involvement in } \\
\text { development of } \\
\text { technological learning } \\
\text { material for clinical skills } \\
\text { training }\end{array}$ & $\begin{array}{c}\text { Cecilie } \\
\text { Haraldseid, } \\
\text { Febe Friberg y } \\
\text { Karina Aase }\end{array}$ & BMC Nursing & $\begin{array}{c}\text { Estudio } \\
\text { exploratorio }\end{array}$ & $\begin{array}{l}\text { Noruega- } \\
\text { Europa }\end{array}$ & Q1 & 7 \\
\hline
\end{tabular}

Finalmente, describiremos el acoplamiento bibliográfico arrojado de los EP. Por medio de la herramienta Vosviewer, se establecieron tres grupos de acoplamiento bibliográfico, aunque tan solo uno presenta conexiones (ver tabla 4). 
Tabla 4. Acoplamiento bibliográfico de las fuentes según la herramienta Vosviewer.

\begin{tabular}{|c|c|}
\hline Grupo & Fuentes relacionadas \\
\hline 1 & $\begin{array}{r}\text { Instructional Science, International Journal of Educational Technology in Higher Education, Assessment \& } \\
\text { Evaluation in Higher Education y Teaching In Higher Education. }\end{array}$ \\
\hline 2 & BMC nursing \\
\hline 3 & Design Studies \\
\hline
\end{tabular}

\subsection{Características de las experiencias de co-diseño de los EP}

Para comenzar, se sintetizan la manera en que se llevaron a cabo los CE en los EP:

1. Doyle et al. (2019) co-diseñaron la evaluación final con sus alumnos (tarea equivalente al $5 \%$ de la calificación del curso). Para ello, en grupos diseñaron de 10-12 preguntas de selección múltiple sobre el tema de la asignatura que les fue asignado. El docente corrigió las preguntas para, luego, compartirlas en el aula virtual de la asignatura. No obstante, solo cinco fueron incluidas en el examen final.

2. Blau y Shamir-Inbal (2018) co-diseñaron el contenido del curso de una asignatura de máster en educación en una universidad israelí mediante la co-creación de la guía didáctica del curso y su diseño pedagógico. Para ello, se realizaron tareas y discusiones apoyadas en las aplicaciones de educación de Google y la herramienta de videoconferencia ZOOM. Por último, se realizó la evaluación mediante la autorreflexión, la retroalimentación entre compañeros del mismo grupo y los demás grupos y el diálogo continuo acerca del contenido del curso.

3. Deeley y Bovill (2017) co-crearon en alianza con sus veintisiete alumnos, mediante el correo electrónico, tres tareas: (1) título de ensayos, (2) criterios de evaluación para la evaluación sumativa del ensayo y (3) para la evaluación sumativa y formativa de un curso de máster en ciencias sociales en una universidad escocesa. Además, los estudiantes se auto-evaluaron y realizaron co-retroalimentación a sus compañeros con los criterios co-diseñados por medio de Äropa.

4. Gros y López (2016) co-diseñaron con once alumnos y seis profesores de dos universidades (Universidad de Barcelona y Universidad Abierta de Cataluña) en cuatro cursos de diferentes disciplinas (Ingeniería biomédica, ciencias económicas, turismo y comunicación) los recursos digitales de sus asignaturas. Para ello, se basaron en el modelo Learner Centric Ecology of Resources (modelo que adapta la teoría socio-cultural de Vygotsky), la enseñanza reflexiva y el aprendizaje potenciado por tecnología.

5. Dorta et al. (2016) co-diseñaron con 14 alumnos de la Universidad de Montreal, dos instituciones industriales internacionales (Withheld - empresa americana de automóviles y 
Design Innovation - oficina de diseño italiana), dos aliados académicos de la universidad de Lorraine (Francia) y cuatro arquitectos egresados de la universidad de Nancy (Francia) que participaron como asesores. El modelo de diseño de Lebahar del área de ingeniería fue adaptando para co-diseñar proyectos cuyo tema era "estructuras livianas que compensen los impactos en automóviles". Cada estudiante tenía una pareja a la que debía apoyar en su proyecto. Semanalmente, tenían sesiones sincrónicas donde discutían sus proyectos con sus profesores y, ocasionalmente, con un colaborador de la industria o académico, en el ambiente inmerso para el desarrollo del co-diseño de la institución.

6. Haraldseid et al. (2016) co-diseñaron con sus alumnos (165) material tecnológico para la asignatura "Entrenamiento de habilidades clínicas en enfermería" en una universidad noruega. El co-diseño se estrucutró en cinco etapas: fase inicial, fase de investigación, fase de revisión, fase de prueba exploratoria y fase final. Los estudiantes contribuyeron en todas las etapas mediante actividades, entrevistas y recolección de datos.

Seguidamente, en la tabla 5, se resumen los resultados de las características de los CE donde se aprecia la tendencia a co-diseñar exclusivamente entre docente y alumnado, a excepción del artículo 5, que incluye profesionales en el área. Asimismo, se observa disparidad en la cantidad de personas involucradas: el 33\% de los estudios superan los 150 participantes (art. 1 y 6), mientras que el 66\% tienen una participación inferior a 55 pero mayor que 17 (art. 2, 3, 4 y 5). En contraste, se examinaron los resultados de la RSL de Mercer-Mapstone et al. (2017) sobre "students as partners" en ES, donde identificamos que tan solo el 7\% involucra a más de 100 alumnos en la asociación entre profesor y estudiante, evidenciando una de las características diferenciadoras entre "students as partners" y CE: la cantidad de alumnos involucrados en las iniciativas. 
Tabla 5. Datos arrojados de las categorías de análisis: tipo de interacción, personas involucradas en CE, institución, tipo de asignatura, de producto, de actividad y de tecnología.

\begin{tabular}{|c|c|c|c|c|c|c|c|}
\hline Artículo & $\begin{array}{c}\text { Tipo de } \\
\text { interacción }\end{array}$ & $\begin{array}{c}\text { Personas } \\
\text { involucradas }\end{array}$ & Institución & $\begin{array}{c}\text { Tipo de } \\
\text { asignatura }\end{array}$ & $\begin{array}{l}\text { Tipo de producto } \\
\text { (A) Tecnológico } \\
\text { (B) No tecnológico } \\
\text { (C) Evaluativo y } \\
\text { retroalimentación } \\
\text { (D) Contenido o } \\
\text { materiales para el } \\
\text { currículo }\end{array}$ & $\begin{array}{l}\text { Tipo de } \\
\text { actividad }\end{array}$ & Tipo de tecnología \\
\hline 1 & $\begin{array}{c}\text { Estudiante- } \\
\text { profesor }\end{array}$ & 162 & Universidad & $\begin{array}{l}\text { Módulo de } \\
\text { impuestos }\end{array}$ & $\begin{array}{l}\text { Evaluación } \\
{[(B) \text { y }(C)]}\end{array}$ & Tareas & $\begin{array}{l}\text { Entorno virtual de } \\
\text { aprendizaje } \\
\text { Correo electrónico }\end{array}$ \\
\hline 2 & $\begin{array}{c}\text { Estudiante- } \\
\text { profesor }\end{array}$ & 54 & Universidad & $\begin{array}{l}\text { Asignatura de } \\
\text { máster en } \\
\text { educación }\end{array}$ & $\begin{array}{l}\text { Contenido del } \\
\text { curso } \\
{[(B),(C) \text { y }(D)]}\end{array}$ & $\begin{array}{l}\text { Discusiones } \\
\text { Tareas }\end{array}$ & $\begin{array}{c}\text { Aplicación de } \\
\text { educación de } \\
\text { Google } \\
\text { Sistema de } \\
\text { videoconferencia - } \\
\text { ZOOM }\end{array}$ \\
\hline 3 & $\begin{array}{c}\text { Estudiante- } \\
\text { profesor }\end{array}$ & 28 & Universidad & Política Pública & $\begin{array}{l}\text { Evaluación } \\
\text { Retroalimentación } \\
\text { [(B) y (D)] }\end{array}$ & $\begin{array}{c}\text { Tareas } \\
\text { Auto- } \\
\text { evaluación } \\
\text { Co- } \\
\text { evaluación }\end{array}$ & $\begin{array}{c}\text { Correo electrónico } \\
\text { Aropä }\end{array}$ \\
\hline 4 & $\begin{array}{c}\text { Estudiante- } \\
\text { profesor }\end{array}$ & 17 & Universidad & $\begin{array}{l}\text { Ingeniería } \\
\text { biomédica } \\
\text { Ciencias } \\
\text { económicas } \\
\text { Turismo } \\
\text { Comunicación }\end{array}$ & $\begin{array}{l}\text { Selección de } \\
\text { recursos digitales } \\
\text { para su curso } \\
{[(B) \text { y (D)] }}\end{array}$ & Taller & $\begin{array}{c}\text { Entorno virtual de } \\
\text { aprendizaje } \\
\text { Video-chat } \\
\text { Skype } \\
\text { Wiki } \\
\text { Facebook }\end{array}$ \\
\hline 5 & $\begin{array}{l}\text { Estudiante - } \\
\text { profesor- } \\
\text { empresa } \\
\text { privada - } \\
\text { aliados } \\
\text { académicos }\end{array}$ & 25 & Universidad & Design studio & $\begin{array}{c}\text { Desarrollar un } \\
\text { diseño } \\
{[(\mathrm{A})]}\end{array}$ & Discusiones & $\begin{array}{l}\text { Augmented Co- } \\
\text { design Studio } \\
\text { Pen-tablet-device } \\
\text { Anamorphic and } \\
\text { 2D visualizations } \\
\text { Hyve-3D } \\
\text { Stop-motion } \\
\text { animations } \\
\text { 2D images } \\
\text { 3D CAD models } \\
\text { 4D-360 immersive } \\
\text { video } \\
\text { 3D printed models }\end{array}$ \\
\hline 6 & $\begin{array}{c}\text { Estudiante- } \\
\text { profesor }\end{array}$ & 170 & Universidad & $\begin{array}{c}\text { Entrenamiento } \\
\text { de habilidades } \\
\text { clínicas en } \\
\text { enfermería }\end{array}$ & $\begin{array}{l}\text { Desarrollar } \\
\text { material } \\
\text { tecnológico } \\
{[(A) \text { y }(D)]}\end{array}$ & $\begin{array}{l}\text { Tareas } \\
\text { Recolecció } \\
\mathrm{n} \text { de datos } \\
\text { Entrevistas }\end{array}$ & $\begin{array}{c}\text { Pad Tablet } \\
\text { Correo electrónico } \\
\text { Foros } \\
\text { Videos } \\
\text { Grabación de } \\
\text { audios }\end{array}$ \\
\hline
\end{tabular}


La rama del conocimiento predominante en el CE, según los EP, son las ciencias sociales (55\%), seguida de las ingenierías y arquitectura (36\%) y, por último, las ciencias de la salud (9\%). Respecto al tipo de producto elaborado, se observa, por un lado, que estos pueden ser tecnológicos (Dorta et al., 2016; Haraldseid et al., 2016) o no tecnológicos (Blau y Shamir-Inbal, 2018; Deeley y Bovill, 2017; Doyle et al., 2019; Gros y López, 2016). Y por otro, que pueden enfocarse en la evaluación y/o retroalimentación (Blau y Shamir-Inbal, 2018; Doyle et al., 2019) o al desarrollo del contenido de un curso o materiales para el mismo (Blau \& Shamir-Inbal, 2018; Deeley \& Bovill, 2017; Gros \& López, 2016; Haraldseid et al., 2016). Respecto al tipo de actividades escogidas para co-diseñar, se destacan la asignación de tareas específicas (Blau y Shamir-Inbal, 2018; Deeley y Bovill, 2017; Doyle et al., 2019; Haraldseid et al., 2016), que dan como resultado el producto co-diseñado, seguidas de las discusiones, debates realizados entre profesores-alumnos (Blau y Shamir-Inbal, 2018), e incluso con aliados externos (Dorta et al., 2016). Finalmente, se diferencian tres tipos de tecnología usada durante el CE. En la tabla 6, se observan las dos herramientas y/o recursos usados para proveer de información a los estudiantes (8\%), las seis seleccionadas para facilitar la comunicación entre los participantes de la experiencia educativa (24\%), y las diecisiete herramientas utilizadas para desarrollar el producto final del codiseño (68\%).

Tabla 6. Tipo de tecnología según funcionalidad

\begin{tabular}{|c|c|}
\hline \multicolumn{2}{|c|}{ Tipo de tecnología } \\
\hline $\begin{array}{c}\text { Para proveer de información a los } \\
\text { estudiantes }\end{array}$ & $\begin{array}{c}\text { Entorno virtual de aprendizaje } \\
\text { Videos }\end{array}$ \\
\hline $\begin{array}{c}\text { Para facilitar la comunicación entre } \\
\text { participantes durante la experiencia } \\
\text { educativa }\end{array}$ & $\begin{array}{c}\text { Entorno virtual de aprendizaje } \\
\text { Correo electrónico } \\
\text { Video-chat } \\
\text { Skype } \\
\text { Facebook } \\
\text { Foros }\end{array}$ \\
\hline $\begin{array}{c}\text { Herramientas necesarias para } \\
\text { desarrollar el producto } \\
\text { co-diseño }\end{array}$ & $\begin{array}{c}\text { Aplicación de educación de Google } \\
\text { Sistema de videoconferencia - ZOOM } \\
\text { Aropä } \\
\text { Wiki } \\
\text { Facebook } \\
\text { Documentos compartidos en línea } \\
\text { Augmented Co-design Studio } \\
\text { Pen-tablet-device } \\
\text { Anamorphic and 2D visualizations } \\
\text { Hyve-3D } \\
\text { Stop-motion animations } \\
\text { 2D images } \\
\text { 3D CAD models } \\
\text { 4D- 360 immersive video } \\
\text { 3D printed models } \\
\text { Pad Tablet } \\
\text { Grabación de audios }\end{array}$ \\
\hline
\end{tabular}




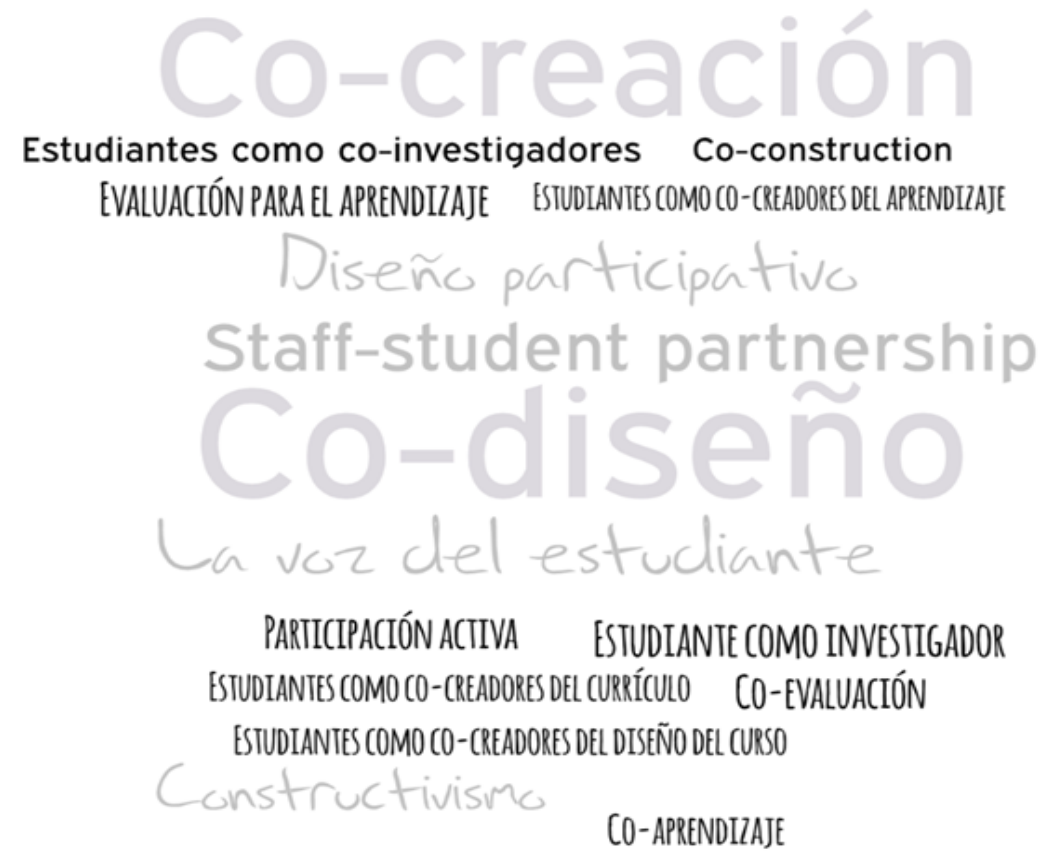

Figura 3. Palabras relacionadas con el concepto de CE

Respecto a las palabras estrictamente vinculadas al concepto de CE según los EP, la más repetida por los autores ha sido el concepto de co-creación en cinco ocasiones, seguido de diseño participativo, la voz del estudiante, "staff-student partnership" y constructivismo, repetidos dos veces, mientras los nueve conceptos restantes, resaltados en negrita en la figura 3 , se han mencionado en una sola ocasión.

Es congruente que estas palabras estén relacionadas puesto que, por un lado, el co-diseño y la coevaluación son diferentes formas de hacer diseño participativo, cuyo resultado puede ser la coproducción o la co-creación (Dollinger et al., 2019). Además, esta última se entiende como una técnica pedagógica que ejemplifica el constructivismo, cuya base es la alianza entre el profesor y el alumno (staff-student-partenership) (Doyle et al., 2019). Además, Púñez (2015, p. 89) considera la evaluación para el aprendizaje otra forma de establecer un proceso participativo inclusivo. Por otro lado, cabe resaltar que existen varias formas de escuchar la voz del estudiante y sumergir al mismo en una participación activa por medio de un diseño de enfoques de enseñanza, cursos y/o planes de estudio (Bovill et al., 2011). Así mismo, en la tabla 7, podemos asociar algunos de estos conceptos a tres áreas de asociación en educación de las cuatro identificados por Healey et al. (2014, p. 36) 
Tabla 7. Relación de los conceptos relacionados con las tres de las áreas de asociación en educación propuestas por Healey et al. (2014, p.36)

\begin{tabular}{|l|l|}
\hline Áreas de asociación en educación & Conceptos relacionados con co-diseño de los EP \\
\hline Aprendizaje, enseñanza y evaluación & $\begin{array}{l}\text { co-aprendizaje, co-evaluación, estudiantes como co-creadores del } \\
\text { aprendizaje, estudiantes como co-creadores del diseño del curso, co- } \\
\text { construcción }\end{array}$ \\
\hline Conocimiento basado en investigación & estudiantes como co-creadores del currículo \\
\hline Diseño del currículo y asesoría pedagógica & estudiantes como investigadores \\
\hline
\end{tabular}

En concordancia, los autores asocian cuatro de estos conceptos con sus co-diseños: el constructivismo fue usado como base teórica para co-diseñar (Doyle et al., 2019), mientras la cocreación (Blau y Shamir-Inbal, 2018; Deeley y Bovill, 2017), la evaluación formativa (Deeley y Bovill, 2017) y el diseño participativo (Haraldseid et al., 2016) como estrategia didáctica. No obstante, se aplicaron otras metodologías y estrategias didácticas, tal como se observa en la figura 4, destacando la elección de "aprendizaje ubicuo" y "aprendizaje multimedia" (Blau y Shamir-Inbal, 2018), y "aprendizaje potenciado por la tecnología" (Gros y López, 2016), ya que fomentan el uso de las TIC en el CE.

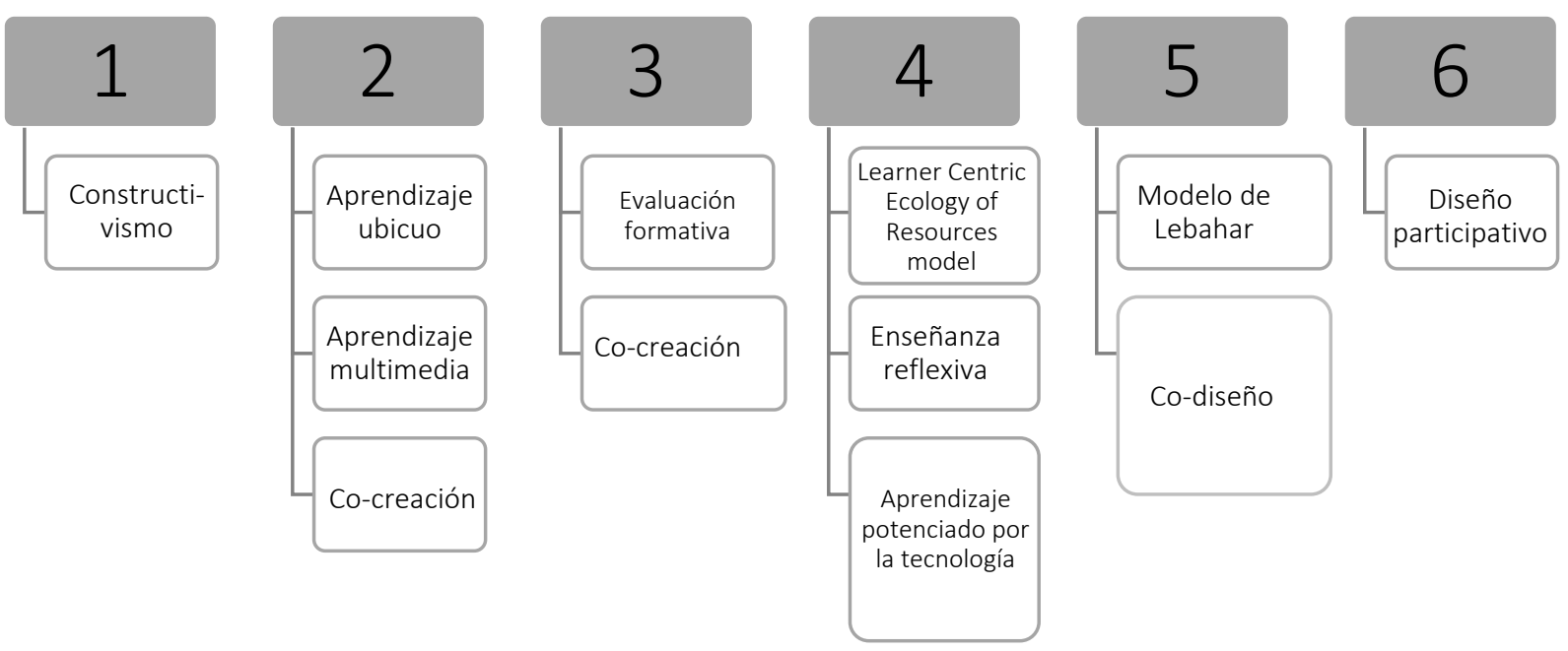

Figura 4. Teorías de aprendizaje y estrategia didáctica usada para el CE en cada estudio. 


\subsection{Beneficios y desafíos presentados durante el CE haciendo uso de las TIC en ES}

A continuación, se presenta, en primer lugar, los beneficios reportados de los estudios y, seguidamente, los desafíos.

Los beneficios, presentados en el anexo 1, están asociados mayormente con las ganancias que el CE brinda a los estudiantes. Para comenzar, se observa la mejora del desempeño académico de los alumnos, quedando demostrado en la calidad e innovación de sus trabajos (Blau y Shamir-Inbal, 2018). Esta mejora está asociada, a su vez, con la mejora del diseño de aprendizaje que surge de la alianza entre profesor y alumno, donde se valora la importancia que cada agente tiene (Haraldseid et al., 2016), convirtiéndose así en un diseño innovador (Deeley y Bovill, 2017). De ahí que, los estudiantes consideren que la experiencia es beneficiosa (Haraldseid et al., 2016) y exitosa para su proceso de aprendizaje (Gros y López, 2016).

Asimismo, la capacidad que demuestran los docentes a la hora de adaptarse a las necesidades de los estudiantes (Deeley y Bovill, 2017), hace que estos se sientan escuchados (Dorta et al., 2016) y valorados, haciéndolos sentir seguros (Deeley y Bovill, 2017). Consecuentemente, se sienten, por un lado, capaces y motivados al tener influencia y control sobre su propio aprendizaje (Deeley y Bovill, 2017; Gros y López, 2016) y, por otro, sienten que el rol del profesor es vital debido al apoyo que este les brinda mediante orientación (Deeley y Bovill, 2017), direccionamiento y retroalimentación constante (Haraldseid et al., 2016). Todo ello haciendo que la clase se convierta en un ambiente inclusivo, creando de esta manera, un sentimiento de comunidad de aprendizaje (Deeley y Bovill, 2017; Dorta et al., 2016) donde la responsabilidad es compartida (Doyle et al., 2019; Gros y López, 2016).

Por otro lado, la participación activa en el CE es un beneficio notorio (Blau y Shamir-Inbal, 2018; Deeley y Bovill, 2017; Gros y López, 2016), ya que los estudiantes se convierten en creadores activos del contenido de la asignatura (Doyle et al., 2019). Asimismo, los alumnos consideran que gracias a esta participación su aprendizaje es significativo (Blau y Shamir-Inbal, 2018; Deeley y Bovill, 2017), puesto que este proceso implica aprender de sus compañeros y auto-reflexionar sobre sus propias prácticas (Deeley y Bovill, 2017). Igualmente, los alumnos relacionan el aprendizaje significativo con la utilidad de la actividad que estén creando, permitiéndoles disfrutar de la tarea y, manteniéndolos motivados y comprometidos en el CE (Doyle et al., 2019; Deeley y Bovill, 2017). También, valoraron positivamente que las tareas fuesen cortas, prácticas, originales, creativas e interesantes (Doyle et al., 2019), así como la necesidad de investigar para realizarlas (Doyle et al., 2019; Gros y López, 2016; Haraldseid et al., 2016).

Igualmente, los estudios analizados muestran que, los estudiantes han comprendido mejor la asignatura (Doyle et al., 2019; Gros y López, 2016; Haraldseid et al., 2016), gracias al trabajo autónomo que el CE conlleva (Deeley y Bovill, 2017; Gros y López, 2016), viéndose beneficiado su desarrollo profesional (Blau y Shamir-Inbal, 2018; Dorta et al., 2016; Gros y López, 2016), y a la adquisición de conocimiento tecnológico y tecno-pedagógico (Blau y Shamir-Inbal, 2018). Igualmente, asocian el CE con la necesidad de hacerse responsables de su aprendizaje (Blau y 
Shamir-Inbal, 2018; Deeley y Bovill, 2017; Gros y López, 2016), teniendo que desarrollar estrategias de autoaprendizaje y habilidades de manejo de su propio proceso formativo (Blau y Shamir-Inbal, 2018; Haraldseid et al., 2016).Finalmente, se considera que la interacción social enfocada al contenido también ha sido beneficiosa para comprender mejor la asignatura, favoreciendo el desarrollo de estrategias tanto socio-emocionales, como aquellas asociadas al aprendizaje colaborativo (Blau y Shamir-Inbal, 2018; Haraldseid et al., 2016).

En segundo lugar, se analizaron los desafíos, representados en el anexo 2, donde se observaron dos tendencias; una inclinada a dar a conocer cuáles son las limitaciones presentadas en los EP y otra encaminada a resaltar cuáles son los requisitos necesarios para que el CE sea pertinente.

Algunos estudios aluden a limitaciones relacionados con obstáculos sentidos por los alumnos. El estudio de Deleey y Bovil (2017) reporta que algunos alumnos sintieron desconfianza e incertidumbre frente al proceso de co-diseño. Los trabajos de Doyle et al. (2019) y Haraldseid et al. (2016) refieren a la necesidad de una retroalimentación continua por parte del profesor que les dé seguridad durante el CE, debido a que algunos alumnos sienten desconfianza cuando reciben retroalimentación por parte de sus compañeros. Paralelamente, Haraldseid et al. (2016), advierte que algunos estudiantes se sienten poco capacitados en el momento de co-diseñar, es decir, sienten que no se tienen las herramientas para aportar en el proceso educativo.

Por otra parte, las carencias presentadas en casos específicos durante el CE se vinculan, por un lado, a la falta de motivación y compromiso que algunos estudiantes muestran, debido a la desconfianza que produce la alianza entre profesor y estudiante, así como la falta de trabajo de los mismos (Deeley y Bovill, 2017) y, por otro, a la falta de equidad en esta alianza presentes en algunos estudios (Deeley y Bovill, 2017; Doyle et al., 2019), debido a la incapacidad de adaptación que algunos participantes tienen frente a las adversidades (Deeley y Bovill, 2017; Gros y López, 2016), conllevando a caer en roles tradicionales de profesor y estudiante como en el estudio de Gros y López, (2016).

Además, en algunas ocasiones, el CE genera ansiedad y estrés a ambos participantes. En el estudio de Haraldseid et al. (2016), se observa que al profesor le afecta la reducción de autoridad mientras que en el estudio de Gros y López (2016), preocupa el tiempo de trabajo que el CE conlleva. Por otro lado, los estudios primarios asocian los siguientes factores a ese estado de ansiedad que algunos estudiantes presentan durante el co-diseño: la falta de estrategias para el trabajo colaborativo, la participación activa, la sobrecarga de información (Blau y Shamir-Inbal, 2018) y de trabajo (Deeley y Bovill, 2017; Doyle et al., 2019), la carencia de recursos (Haraldseid et al., 2016), el uso de las TIC (Blau y Shamir-Inbal, 2018; Deeley y Bovill, 2017; Gros y López, 2016) y el hecho de profundizar en algunos temas específicos del temario (Doyle et al., 2019; Gros y López, 2016; Haraldseid et al., 2016). Otro aspecto a mejorar en el CE, que también genera ansiedad (Deeley y Bovill, 2017; Haraldseid et al., 2016) y estrés en el alumnado (Blau y Shamir-Inbal, 2018; Dorta et al., 2016), es el rol del profesor como guía (Gros y López, 2016; Haraldseid et al., 2016), asociado 
a la falta de comunicación asertiva (Haraldseid et al., 2016), de instrucción (Doyle et al., 2019) y de retroalimentación (Doyle et al., 2019; Haraldseid et al., 2016).

Por otro lado, se considera requisitos indispensables para el CE generar un cambio epistemológico (Blau y Shamir-Inbal, 2018; Deeley y Bovill, 2017; Gros y López, 2016), que conlleve tanto a cambios operacionales (Doyle et al., 2019) como de hábitos de aprendizaje (Blau y Shamir-Inbal, 2018), siendo imprescindible la capacidad de adaptación tanto de profesores como estudiantes (Deeley y Bovill, 2017; Gros y López, 2016). Para ello, es fundamental que se mantenga una participación activa durante el CE, estableciendo una base sólida de confianza entre los participantes (Blau y Shamir-Inbal, 2018). Asimismo, el CE requiere que el estudiante desarrolle, por un lado, su autonomía (Haraldseid et al., 2016), debido al nivel de disciplina y planeación que este proceso demanda y, por otro, el aprendizaje colaborativo, que exige a su vez, habilidades de pensamiento socio-emocional (Blau y Shamir-Inbal, 2018).

\section{DISCUSIÓN Y CONCLUSIONES}

En este artículo se ha identificado la manera en que los estudiantes y profesores co-diseñan haciendo uso de las TIC conforme con la literatura científica de los seis últimos años. Primero, se determinaron las características biliométricas de los EP, concluyendo que existe una limitada bibliografía (seis EP) que abarque el objeto de estudio, debido a los criterios de inclusión: tipo de co-diseño (educativo), contexto (ES) y, especialmente, uso de las TIC, entendiéndose como la mayor limitación de este estudio. No obstante, los resultados concernientes a las características de las experiencias del CE y a los beneficios y desafíos que estos arrojan, denotan relevancia, ya que contribuyen al conocimiento científico.

El CE se entiende como una oportunidad de innovación docente donde los estudiantes se involucran de forma exigente y productiva (González et al., 2016) y cuyo proceso adquiere valor tanto para el profesor como para los estudiantes (Dollinger y Lodge, 2019), ya que ambos participantes perciben beneficios de empleabilidad y autoeficacia en su desempeño (Dollinger y Lodge, 2019). Otro de los beneficios que derivan de este tipo de asociación es el aumento de la conciencia meta-cognitiva para ambos participantes, que está directamente relacionado con la mejora del aprendizaje y las experiencias dentro del aula (Felten et al., 2014), puesto que el CE requiere de estrategias de autoaprendizaje y autogestión. Además, estas estrategias requieren a su vez de "estrategias cognitivas de pensamiento; estrategias de apoyo para el autocontrol del esfuerzo, meta-cognitivas y motivacionales, que permiten el aprendizaje significativo"(González Fernández et al., 2018, p.18). En definitiva, el co-diseño educativo es una de las estrategias educativas del siglo XXI que busca trabajar de manera colaborativa con sus alumnos con el fin de empoderarlos en su proceso educativo, fomentando de esta manera el aprendizaje significativo que conlleva una participación activa constante entre ambos participantes (profesores $y$ estudiantes). 
Asimismo, el CE, al fomentar la participación activa, ofrece al estudiante la capacidad de decidir e influir en su propio proceso educativo (González et al., 2016). Además, la alianza que emana de este proceso mejora tanto la motivación como el aprendizaje de ambos participantes, ya que, por un lado, aumenta la confianza del estudiante, que se enfoca en su proceso educativo en lugar del producto final, y por otro, ayuda al docente a repensar el proceso de enseñanza (Felten et al., 2014). Sin embargo, se ha observado que el co-diseño desafía las relaciones de poder tradicionales entre profesor y estudiante, que pueden entenderse como una amenaza a la identidad personal y profesional, generando resistencia a probar nuevos métodos por parte de los participantes (Healey et al. (2014). Del mismo modo, Deeley y Bovill, (2017) admiten que este tipo de alianza no siempre es apropiada, específicamente en el área de evaluación de la asignatura.

Por ello, si se desea co-diseñar, se hace necesario que se establezca una base sólida de confianza entre el profesor y el estudiante, que lleve a un buen entendimiento entre ambas partes (Brown, 2019). Además, se debe trabajar la comunicación entre los participantes ya que se considera un aspecto clave para el efectivo desarrollo del CE (Bovill et al., 2016).Aquellos académicos que deciden trabajar de manera colaborativa con sus alumnos deben ser conscientes de su papel como facilitador (Brown, 2019) y comprender su rol como guía, además de cerciorarse del nivel de participación que deciden asumir con sus estudiantes en la co-creación (Kaminskiene et al., 2020). Para Brown, (2019), el trabajo colaborativo y la efectividad del mismo está influenciado tanto por el rol del instructor y su posición, como por los niveles de dificultad, el diseño de la tarea a realizar y los resultados esperados de este proceso. De ahí, la importancia de seleccionar actividades de enseñanza-aprendizaje a co-crear que sean motivadoras y ricas para ambos participantes (González et al., 2016). Además, se hace necesario que el docente establezca un marco claro donde todos los interesados puedan trabajar apropiadamente (Brown, 2019). Por ello, las sesiones deben estar organizadas rigurosamente, los objetivos deben ser explícitos y asequibles y el trabajo cooperativo entre las partes debe estar garantizado (González et al., 2016).

Finalmente, se observa que, aunque el CE presenta algunos desafíos, son estos mismos los que hacen beneficiosa la asociación entre profesor y docente. Además, se sugiere que para el buen funcionamiento del CE, se cumplan unos requisitos específicos. Futuros estudios, podrían encaminarse hacia el diseño de un modelo para el co-diseño educativo mediado por tecnología educativa que establezca secuencias didácticas, así como, la indagación acerca de cómo co-diseñar entre profesor y estudiante en el área de evaluación apropiadamente haciendo uso de las TIC.

\section{REFERENCIAS}

Blau, I. y Shamir-Inbal, T. (2018). Digital technologies for promoting "student voice" and co-creating learning experience in an academic course. Instructional Science, 46(2), 315-336. https://doi.org/10.1007/s11251-017-9436-y

Bovill, C., Cook-Sather, A., Felten, P., Millard, L. y Moore-Cherry, N. (2016). Addressing potential DOI: https://doi.org/10.21556/edutec.2020.74.1799

Página 45 / 50 
challenges in co-creating learning and teaching: overcoming resistance, navigating institutional norms and ensuring inclusivity in student-staff partnerships. Higher Education, 71(2), 195-208. https://doi.org/10.1007/s10734-015-9896-4

Bovill, C., Cook-Sather, A. y Felten, P. (2011). Students as co-creators of teaching approaches, course design, and curricula: Implications for academic developers. International Journal for Academic Development, 16(2), 133-145. https://doi.org/10.1080/1360144X.2011.568690

Bovill, C. y Bulley, C. J. (2011). A model of active student participation in curriculum design: exploring desirability and possibility. Improving Student Learning (ISL) 18: Global Theories and Local Practices: Institutional, Disciplinary and Cultural Variations., July, 176-188.

Brown, N. (2019). Partnership in learning: how staff-student collaboration can innovate teaching. European Journal of Teacher Education, 42(5), 608-620. https://doi.org/10.1080/02619768.2019.1652905

Bryson, C. (2016). Engagement through partnership: students as partners in learning and teaching in higher education. International Journal for Academic Development, 21(1), 84-86. https://doi.org/10.1080/1360144x.2016.1124966

Deeley, S. J. y Bovill, C. (2017). Staff student partnership in assessment: enhancing assessment literacy through democratic practices. Assessment and Evaluation in Higher Education, 42(3), 463-477. https://doi.org/10.1080/02602938.2015.1126551

Dollinger, M., Liu, D., Arthars, N. y Lodge, J. M. (2019). Working together in learning analytics towards the co-creation of value. Journal of Learning Analytics, 6(2), 10-26. https://doi.org/10.18608/ila.2019.62.2

Dollinger, M., \& Lodge, J. (2019). Student-staff co-creation in higher education: an evidence-informed model to support future design and implementation. Journal of Higher Education Policy and Management, 42(5), 532-546. https://doi.org/10.1080/1360080X.2019.1663681

Dorta, T., Kinayoglu, G. y Boudhraâ, S. (2016). A new representational ecosystem for design teaching in the studio. Design Studies, 47, 164-186. https://doi.org/10.1016/j.destud.2016.09.003

Doyle, E., Buckley, P. y Whelan, J. (2019). Assessment co-creation: an exploratory analysis of opportunities and challenges based on student and instructor perspectives. Teaching in Higher Education, 24(6), 739-754. https://doi.org/10.1080/13562517.2018.1498077

Felten, P., Bovill, C. y Cook--Sather, A. (2014). Engaging students as partners in learning and teaching (1): benefits and challenges - what do we know? International Consortium for Educational Development Conference. https://bit.ly/3m3GeCB

González, A. A., Roig, A. E., Suari, N. O. y Juanola, M. M. (2016). Aprendizaje-servicio y codiseño en la formación de maestros: Vías de integración de las experiencias y perspectivas de los estudiantes. Bordon, 68(2), 169-183. https://doi.org/10.13042/Bordon.2016.68211 
González Fernández, M. O., Becerra Vázquez, J. J. y Olmos Cornejo, J. E. (2018). Promoción de la autogestión a través de objetos de aprendizaje adaptativos en alumnos de educación superior. Edutec. Revista Electrónica de Tecnología Educativa, 63. https://doi.org/10.21556/edutec.2018.63.1037

Gros, B. (2019). La investigación sobre el diseño participativo de entornos digitales de aprendizaje. 169. https://bit.ly/2V5zi7e

Gros, B. y López, M. (2016). Students as co-creators of technology-rich learning activities in higher education. International Journal of Educational Technology in Higher Education, 13(1). https://doi.org/10.1186/s41239-016-0026-x

Guirao Goris, S. J. A. (2015). Utilidad y tipos de revisión de literatura. Ene, 9(2), 0-0. https://doi.org/10.4321/s1988-348x2015000200002

Hanni-Vaara, P., Kähkönen, O. y Paloniemi, P. (2019) Developing Destination Experience and Digital Marketing in Co-creation Process - Case Cooperation of Ranua Municipality and Lapland University of Applied Sciences. e-Review of Tourism Research, 17(2). https://ertr-ojstamu.tdl.org/ertr/index.php/ertr/article/view/512

Haraldseid, C., Friberg, F. y Aase, K. (2016). How can students contribute? A qualitative study of active student involvement in development of technological learning material for clinical skills training. BMC Nursing, 15(1), 1-10. https://doi.org/10.1186/s12912-016-0125-y

Healey, M., Flint, A. y Harrington, K. (2014). Engagement through partnership: students as partners in learning and teaching in higher education. https://bit.ly/367/8N9

Jussila, J., Raitanen, J., Partanen, A., Tuomela, V., Siipola, V. y Kunnari, I. (2019). Rapid Product Development in University- Industry Collaboration: Case Study of a Smart Design Project. Technology Innovation Management Review, 10(3). https://bit.ly/2KLz3kV

Kalantzis, M. y Cope, B. (2010). The teacher as designer: Pedagogy in the new media age. E-Learning, 7(3), 200-222. https://doi.org/10.2304/elea.2010.7.3.200

Kaminskiene, L., Žydžiunaite, V., Jurgile, V. y Ponomarenko, T. (2020). Co-creation of learning: A concept analysis. European Journal of Contemporary Education, 9(2), 337-349. https://doi.org/10.13187/ejced.2020.2.337

Könings, K. D., Mordang, S., Smeenk, F. y Stassen, L. (2020). Learner involvement in the co-creation of teaching and learning: AMEE Guide No. 138. Medical Teacher, 0(0), 1-13. https://doi.org/10.1080/0142159X.2020.1838464

Manrique-Losada, B., Zapata Cárdenas, M. I. y Arango Vásquez, S. I. (2020). Entorno virtual para cocrear recursos educativos digitales en la educación superior. Campus Virtuales, 9(1), 101-112. https://bit.ly/376UJPW

Mcfaul, H., Fitzgerald, E., Byrne, D. y Ryan, F. (2020). A mobile app for public legal education : a case 
study of co-designing with students. Research in Learning Technology, 28(1063519), 1-15. https://doi.org/10.25304/rlt.v28.2434

Mercer-mapstone, L., Bovill, C., Mercer-mapstone, L. y Bovill, C. (2019). Studies in Higher Education Equity and diversity in institutional approaches to student - staff partnership schemes in higher education partnership schemes in higher education. Studies in Higher Education, 45(12), 25412557. https://doi.org/10.1080/03075079.2019.1620721

Mercer-Mapstone, L., Dvorakova, S. L., Matthews, K. E., Abbot, S., Cheng, B., Felten, P., Knorr, K., Marquis, E., Shammas, R. y Swaim, K. (2017). A Systematic Literature Review of Students as Partners in Higher Education. International Journal for Students as Partners, 1(1). https://doi.org/10.15173/ijsap.v1i1.3119

Pee, L. G. (2020). Enhancing the learning effectiveness of ill- structured problem solving with online cocreation. Studies in Higher Education, 45(11), 2341-2355. https://doi.org/10.1080/03075079.2019.1609924

Petticrew, M. y Roberts, H. (2006). Systematic Reviews in the Social Sciences: A Practical Guide. Blackwell Publishing. https://doi.org/10.1002/9780470754887

Púñez, M. (2015). Evaluación para el aprendizaje: una propuesta para una cultura evaluativa. Horizonte de la Ciencia, 5(8), 87-96. https://bit.ly/39fMLGC

Sanders, E. B.-N. y Stappers, P. J. (2008). Co-creation and the new landscapes of design. CoDesign, 4(1), 5-18. https://doi.org/10.1080/15710880701875068

Silva, M. y Rossi, F. (2018). Technological Forecasting \& Social Change The e ff ect of fi rms ' relational capabilities on knowledge acquisition and co- creation with universities. Technological Forecasting \& Social Change, 133(May 2017), 72-84. https://doi.org/10.1016/i.techfore.2018.03.004

Treasure-Jones, T., Dent-Spargo, R. y Dharmaratne, S. (2018). How do students want their workplacebased feedback visualized in order to support self-regulated learning? Initial results \& reflections from a co-design study in medical education. CEUR Workshop Proceedings, 2193.

Zawacki-Richter, O., Kerres, M., Bedenlier, S., Bond, M. y Buntins Eds, K. (2020). Systematic Reviews in Educational Research: Methodology, Perspectives and Application. Springer VS. https://doi.org/10.1007/978-3-658-27602-7

\section{Para citar este artículo:}

Santana Martel, J. S., y Perez-i-Garcias, A. (2020). Co-diseño educativo haciendo uso de las TIC en educación superior una revisión sistemática de literatura. Edutec. Revista Electrónica De Tecnología Educativa, (74), 25-50. https://doi.org/10.21556/edutec.2020.74.1799 


\section{Anexos}

Anexo 1. Red de códigos sobre los beneficios presentados durante el Co-diseño Educativo haciendo uso de las Tecnologías de la Información y Comunicación en educación superior.

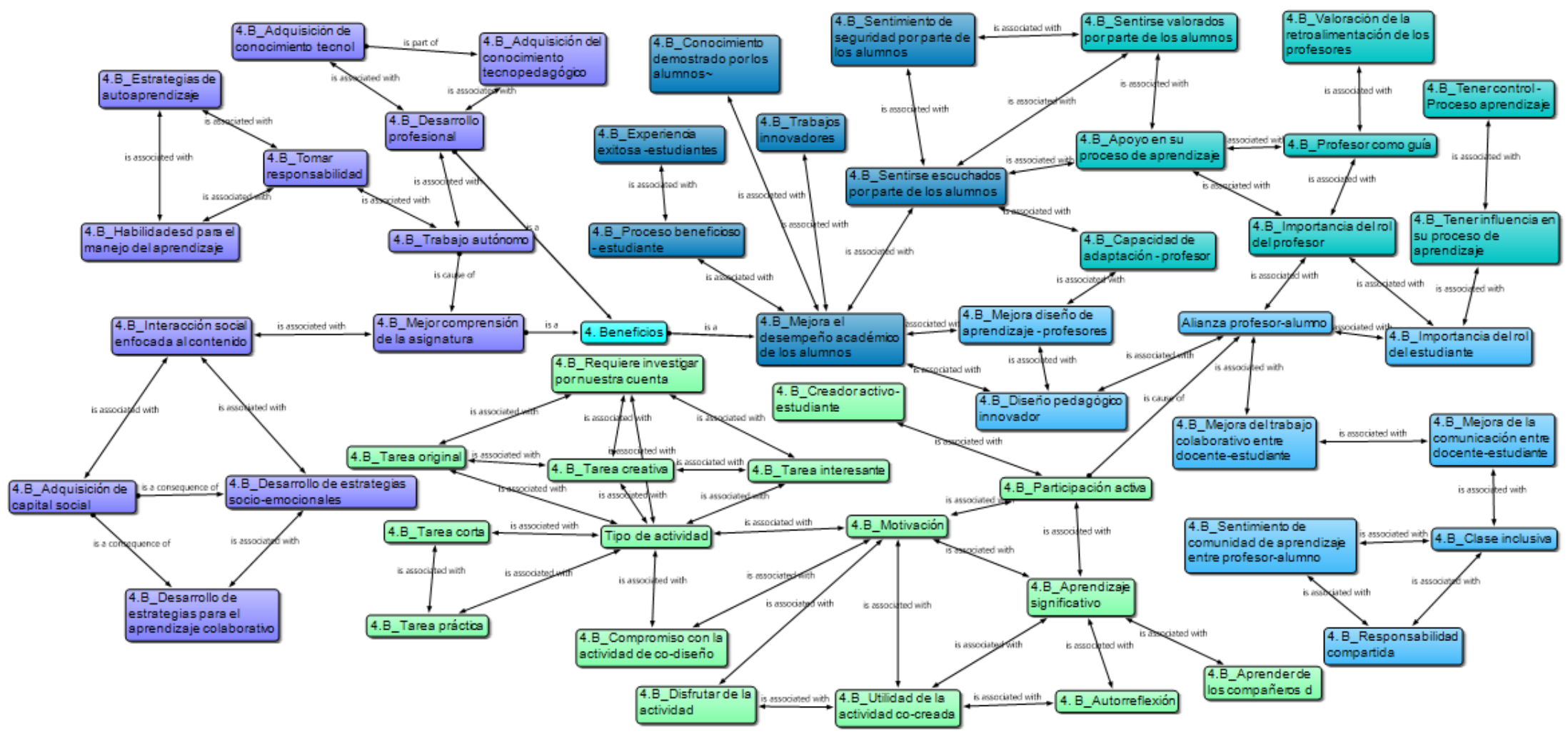


Anexo 2. Red de códigos sobre los desafíos presentados durante el Co-diseño Educativo haciendo uso de las Tecnologías de la Información y Comunicación en educación superior.

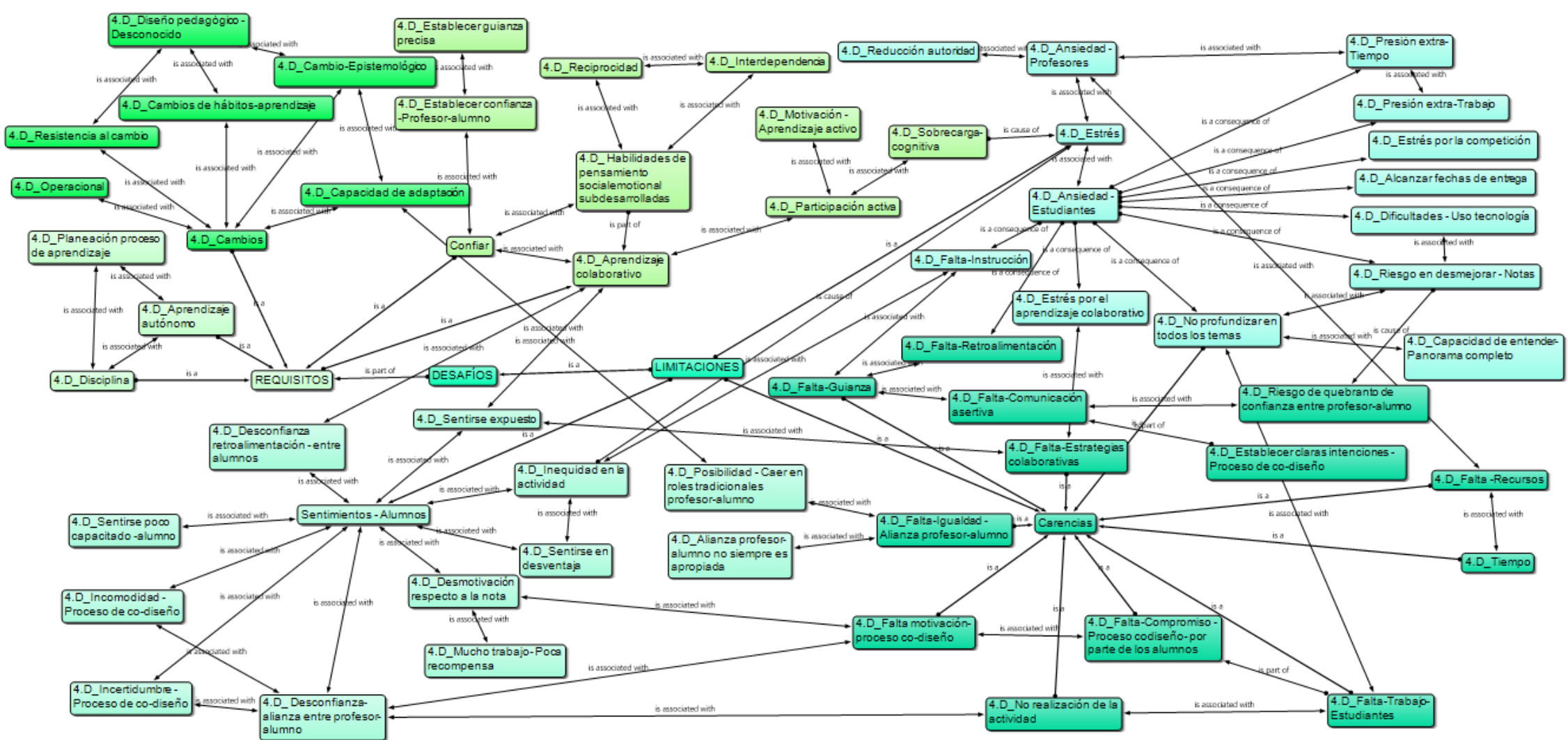

